\title{
Pediatric hypothermic submersion incident - should we do chest compressions on a beating heart?
}

\author{
Steinar Einvik ${ }^{1 *}$, Andreas Jorstad Kruger ${ }^{2,3,4}$ and Sven Erik Gisvold ${ }^{2,4}$
}

\begin{abstract}
Background: Drowning is the third leading cause of unintentional injury death worldwide, with the highest rates of fatality among young children. To decide how to treat these patients prehospitally could be challenging in certain situations when uncertain about the adequacy of the patent's circulation.

Methods/case report: We describe a 2 year old boy surviving a 15 min hypothermic submersion in a cold river. In spite of the presence of some vital signs, we decided to do full cardiopulmonary resuscitation to the hospital. The main reason was that we were uncertain about the adequacy of the spontaneous circulation, and the transport to hospital was fairly long. The patient suffered no obvious harm and the outcome was good.

Discussion: What is regarded as adequate circulation when accidentally hypothermic between 24 and $25^{\circ} \mathrm{C}$ ? A weak pulse was felt in the femoral artery with a rate of about 40-50 per minute. There were shallow, but regular respiration, and point of care ultrasound revealed a slightly dilated left ventricle and weak, but organised cardiac contractions. Despite these findings a decision was made to continue ventilations and chest compressions during helicopter transport to the University hospital.

Conclusion: In an accidentally hypothermic pediatric submersion incident we decided to do full cardiopulmonary resuscitation to the hospital despite there were signs of circulation. We did no harm to the patient. Future guideline revisions should try to clarify how to handle situations with severly accidentally hypothermic patients like this, so the good outcome that is often seen in these patients could be even better.
\end{abstract}

\section{Introduction}

Drowning is the third leading cause of unintentional injury death worldwide, with the highest rates of fatality among young children [1]. Out of hospital it can sometimes be challenging to decide how to treat these patients, but inhospital extracorporeal life support (ECLS) has revolutionized the management of hypothermic cardiac arrest, with greatly improved survival rates [2]. The reason for this is probably the neuroprotective effect of the cooling of the brain. Cerebral metabolic rate for

\footnotetext{
* Correspondence: Steinar.einvik@stolav.no

'Department of Emergency Medicine and Prehospital Services, St. Olav's University Hospital, NO-7006 Trondheim, Norway

Full list of author information is available at the end of the article
}

oxygen $\left(\mathrm{CMRO}_{2}\right)$ is reduced by approximately $8 \%$ per ${ }^{\circ} \mathrm{C}$ fall in temperature. At a core temperature of $28^{\circ} \mathrm{C}$ the $\mathrm{CMRO}_{2}$ and pulse rate is $50 \%$ of the normal state [3].

\section{Case}

We describe a 2 year old, previously healthy boy, who underwent a hypothermic submersion incident in a cold creek close to his home. The outside temperature was $6{ }^{\circ} \mathrm{C}$. It was heavy rainfall and the small creek was like a river at the time of the incident. The boy was not observed when he fell into the river, and his father started searching for him about $10-15$ min later. He was found lifeless caught in the branch of a tree $80 \mathrm{~m}$ downstream. The boy was evacuated and CPR was started without

(c) The Author(s). 2020 Open Access This article is licensed under a Creative Commons Attribution 4.0 International License, which permits use, sharing, adaptation, distribution and reproduction in any medium or format, as long as you give appropriate credit to the original author(s) and the source, provide a link to the Creative Commons licence, and indicate if changes were made. The images or other third party material in this article are included in the article's Creative Commons licence, unless indicated otherwise in a credit line to the material. If material is not included in the article's Creative Commons licence and your intended use is not permitted by statutory regulation or exceeds the permitted use, you will need to obtain permission directly from the copyright holder. To view a copy of this licence, visit http://creativecommons.org/licenses/by/4.0/. The Creative Commons Public Domain Dedication waiver (http://creativecommons.org/publicdomain/zero/1.0/) applies to the data made available in this article, unless otherwise stated in a credit line to the data. 
delay. Shortly thereafter the resuscitation was assisted by a neighbour who was a professional EMS worker.

When the first ambulance arrived on-scene $15 \mathrm{~min}$ later, no breathing nor pulses was detected and CPR was continued. Two air ambulance units were alarmed and dispatched. Meanwhile, assisted breathing was performed by bag, valve and mask ventilation with supplemental oxygen. An intraosseus needle was inserted and epinephrine 150 micrograms every 3 mins was administered. The estimated bodyweight was $16 \mathrm{~kg}$.

The first air ambulance arrived on-scene 20 mins after the start of CPR. The crew detected still no breathing and no pulse and decided to intubate the patient with a cuffed 4.0 ETT without prior medications. The $\mathrm{EtCO}_{2}$ value was between 13 and $16 \mathrm{mmHg}$ on-scene.

Ten to fifteen minutes later (30-35 min after start of CPR) shallow but regular respirations was observed. $\mathrm{EtCO}_{2}$ was still around $13-16 \mathrm{mmHg}$. A weak pulse was felt in the femoral artery with a rate of about $40-50$ per minute. Then narrow-formed ECG complexes were observed on the monitor. The temperature was measured at $24.5^{\circ} \mathrm{C}$ in the upper part of esophagus. Point of care ultrasound revealed a slightly dilated left ventricle and weak, but organised cardiac contractions. It was decided to stop epinephrine boluses, and despite the thready pulse that was felt in the groin it was decided to continue chest compressions in the helicopter during the 28 mins flight to St. Olav's University Hospital in Trondheim. There were no active rewarming during transport to the hospital except the slightly raised temperature held in the cabin. The patient core body temperature was the same at arrival in the hospital as on the incident site.

The patient was transferred directly to the thoracic operation theatre and extracorporeal rewarming was prepared but never used At this time the weak femoral pulse was still palpable and chest compressions were discontinued. An arterial cannula was inserted and the blood pressure (BP) was measured at 110/70. Blood gas revealed a $\mathrm{pH}$ of 6.86 and S-potassium was $2.1 \mathrm{mmol} / \mathrm{l}$. The S-Lactate was slightly above normal. At this time the body temperature measured in the upper oesophagus was $24.6^{0} \mathrm{C}$. The patient was rewarmed using $3 \mathrm{M}^{\mathrm{m}}$ Bair Hugger. Four hours after arrival in the hospital, the core body temperature was $34.5^{\circ} \mathrm{C}$ and active external rewarming was withdrawn.

The patient developed pulmonary oedema during the first hours after arrival. Nitrogenmonoksid (NO) treatment was started, and the condition improved thereafter. $15 \mathrm{~h}$ after arrival at the hospital the patient had normal oxygenation and $\mathrm{FiO}_{2}$ at $30 \%$ with normal respirator settings.

Two days later he was weaned from the respirator, and made a full recovery with no signs of neurological injury.

\section{Discussion}

According to the current guidelines the recommended treatment for hypothermic cardiac arrest includes CPR and transport to a hospital with extracorporeal membrane oxygenation (ECMO) capacity. A good outcome has been reported after 190 mins of CPR during transport to a hospital with extracorporeal rewarming [4]. However, if there is organised cardiac activity, one should start warming the patient externally with heated blankets and warm fluids given intravenously. The treatment protocols have highlighted the importance of treating these patients with extreme caution in order not to trigger a more malignant rhythm like ventricular fibrillation (VF) or ventricular tachycardia (VT) [4].

In this case we faced several challenges. The first was whether we should continue CPR in-flight to the hospital, despite the signs of circulation that we had found on-site. We felt a weak pulse in the groin, and there were regular, though shallow respirations. To help us decide whether the circulation was adequate, we did point of care ultrasound (POCUS) of the heart. We could see organised myocardial contractions, though the left ventricle was dilated, and not acting normal.

The second challenge was whether all these clinical findings were helpful in deciding whether the circulation in this situation should be considered adequate? In fact, the two EMS physicians on site were not certain that the cardiac contractions seen on POCUS were adequate for the child in this situation.

The third challenge was if we decided to continue chest compressions to the hospital, as we did, what was the risk for inducing VF from the patients current narrow-complex perfusing rhythm? Expert opinions [5] argue that agitation of a patient with a temperature of less than $28^{\circ} \mathrm{C}$ carries a significant risk of inducing VF. On the contrary the ERC guidelines 2015 recommend to start CPR immediately if there is any doubt. We were clearly in doubt so we decided to continue CPR to the hospital [6]. The guidelines do not include decision making in situations like we encountered, which can be described as the "peri-arrest" state [5]. Despite all this, the 2015 ERC guidelines told us that when in doubt, you are not in doubt, you should start/continue CPR, and that was what we did.

The decision to continue chest compressions en-route to the hospital was based on the fact that the patient had a low core body temperature, he was not conscious, had shallow respirations and weak myocardial contractions seen on ultrasound.

The question arises whether it is correct to continue CPR during transport in a patient with a palpable pulse and spontaneous respiration. With a temperature below $25^{\circ} \mathrm{C}$ the $\mathrm{CMRO}_{2}$ is reduced with more than $60 \%$. A weak pulse and shallow respiration is perhaps adequate, 
and a "normal" adjustment to the reduced needs of the body in this situation. We had to make a quick decision on-scene and decided to do CPR assuming that oxygen transport was inadequate and needed to be supported by chest compressions and ventilations.

In this case, the patient outcome was good, so at least we did not harm the patient. There were still spontaneous circulation and respiration on arrival at hospital. Our chest compressions and intubation could easily have triggered a change of rhythm to VF or VT. If this had been the case on arrival, the patient had probably been treated with ECMO, a procedure which carries a certain risk. In the Swiss grading system (I-IV) of accidental hypothermia [7] our patient represents stage $\operatorname{III}\left(<28^{\circ} \mathrm{C}\right.$, unconscious, but with vitals present). Due to this staging system the prehospital treatment includes considering transfer to the nearest ECMO centre, and initiate ECMO treatment if signs of cardiac instability. There are published guidelines for indications for ECMO in accidental hypothermia [5]. These include:

1. Prehospital cardiac instability

2. Systolic blood pressure under $90 \mathrm{mmHg}$

3. Ventricular arrhythmias

4. Core temperature $<28^{\circ} \mathrm{C}$

Our patient had 1 and 4 above, but we could not get any measurement of the $\mathrm{BP}$ on-site. At arrival in the hospital the BP was measured at 110/70, as mentioned earlier. The patient was never observed with any ventricular arrhythmias.

The hospital we transported the patient to was not used to treat pediatric patients on ECMO, but are able to provide the treatment in emergencies.

Evaluation of the circulation in deeply hypothermic children is not well described in the litterature. However, in a review by Brown et al. it is stated that detecting a pulse in a hypothermic patient may be very difficult, so signs of life and pulse should be carefully checked for $60 \mathrm{~s}$ [4]. If no signs of life are detected, CPR should be started [4]. Moreover, they state that in patients with a temperature between $24^{\circ} \mathrm{C}$ and $28^{\circ} \mathrm{C}$ and vital signs present, CPR is not necessary. This is contradictory to the expert opinion mentioned earlier [5].

So the question remains; did we do anything wrong and subject the patient to unnecessary risk when treating him like he was in cardiac arrest? The question is difficult to answer. In this case the outcome was beneficial, 4 days after the incident the boy was observed walking around with no detectable neurological sequelae. The favourable outcome might be due to a short submersion time and a prompt CPR performed by witness, whom one of them was by chance a professional.. It is of course possible that the result would have been the same if
CPR had not been performed. A Dutch review of pediatric hypothermic cardiac arrest in children found that good neurological outcome is more likely when return of spontaneous circulation occurs within $30 \mathrm{~min}$ [8]. In their material none of the 12 children receiving prolonged resuscitation( $>30$ mins) after cardiac arrest due to drowning in winter had a good outcome. As such, prognostication as well as clinical decision-making might be dependent on exact timelines. The exact time our patient regained spontaneous circulation is not known.

\section{Conclusion}

In this case report we describe a 2 year old boy surviving a 15 min hypothermic submersion in a river. In spite of the presence of some vital signs, we decided to do full $\mathrm{CPR}$. The main reason was that we were uncertain about the adequacy of the spontaneous circulation, and the transport to hospital was fairly long. The patient suffered no obvious harm and the outcome was good. Future guideline revisions should try to clarify how to handle situations like this, so the good outcome that is often seen in these patients could be even better.

\section{Abbreviations \\ CPR: Cardiopulmonary resuscitation; ECMO: Extracorporeal membrane oxygenation; $\mathrm{CMRO}_{2}$ : Cerebral metabolic rate of oxygene; $\mathrm{EtCO}_{2}$ : End tidal carbondioxide; VF: Ventricular fibrillation; VT: Ventricular tachycardia}

\section{Acknowledgements \\ None. \\ Authors' contributions \\ Corresponding author: Steinar Einvik was one of the two prehospital physicians on the incident and is the primary author of this manuscript. SE finished the manuscript for publication. Co-authors: Andreas Jorstad Kruger has revised and made major contributions to the manuscript. AJK has accepted the final draft for publication. Sven Erik Gisvold has given significant contributions to the manuscript, revised the final draft and accepted the manuscript for publication. The author(s) read and approved the final manuscript}

\section{Authors' informations}

SE is an anestethist and prehospital physician working in Helicopter Emergency Services at St. Olav's University Hospital In Trondheim. He has a special interest for cardiopulmonary resuscitation and prehospital ultrasound. AJK is an anesthetist and prehospital physician working Helicopter Emergency Services at. St. Olav's University Hospital in Trondheim, and as an Associate Professor at Norwegian University of Science and Technology and as a researcher at Norwegian Air Ambulance Foundation. He holds a Ph. D from the theme Physician based Air Ambulance Services from 2012. SEG is an anesthetist and Professor Emeritus in Anaesthesiology from St. Olav's University Hospital and Norwegian University of Science and Technology. He has previously been an Editor-in-Chief of the Acta Anaesthesiologica Scandinavica.

\section{Funding}

Not applicable.

Availability of data and materials Not applicable.

Ethics approval and consent to participate

Do to legislation in Norway the Ethics Comittees do not process single case reports. What is needed is a consent for publication from the patient or the 
parents if the patient is a child, as is the case in this report. For consent from the parents, see the "Consent for publication" section below.

\section{Consent for publication}

We have an attachment from the parents that consent this case report for publication.

\section{Competing interests}

The authors declare that they have no competing interests.

\section{Author details}

'Department of Emergency Medicine and Prehospital Services, St. Olav's University Hospital, NO-7006 Trondheim, Norway. ${ }^{2}$ Department of Anaesthesia and Intensive Care, St. Olav's University Hospital, NO-7006 Trondheim, Norway. ${ }^{3}$ Department of Research and Development, Norwegian Air Ambulance Foundation, NO-0103 Oslo, Norway. ${ }^{4}$ Department of Circulation and Medical Imaging, Norwegian University of Science and Technology, NO-7006 Trondheim, Norway.

Received: 27 May 2020 Accepted: 11 August 2020

Published online: 20 August 2020

\section{References}

1. Kriz D, Piantino J, Fields D, Williams C. Pediatric Hypothermic Submersion Injury and Protective Factors Associated with Optimal Outcome: A Case Report and Literature Review. Children. 2018:5(1):4. Published online 2017 Dec 27. https://doi.org/10.3390/children5010004.

2. Paal P, Gordon L, Strapazzon G, Maeder MB, Putzer G, Walpoth B, Wanscher M, Brown D, Holzer M, Broessner G, Brugger H. Accidental hypothermia-an update. Scand J Trauma Resusc Emerg Med. 2016;24:111. Published online 2016 Sep 15. https://doi.org/10.1186/s13049-016-0303-7.

3. Srilata M, Jayaram K. Chapter 35 Hypothermia; Complications in Neuroanesthesia; 2016. p. 331-44.

4. Brown DJA, Brugger H, Boyd J, Paal P. Accidental hypothermia. Review article. N Engl J Med. 2012;367:1930-8.

5. Emergencymedicinecases.com. Crit Cases 5- Pediatric drowning and Hypothermia; Expert opinion; 2016. p. 8. Michael Misch.

6. Truhlar A, et al. European Resuscitation Council Guidelines for Resuscitation 2015: Section 4. Cardiac arrest in special circumstances.

7. Cline DM, Ma OJ, Meckler GD, Tintinalli JE, Stapczynski JS, Yealy D. Tintinalli's Emergency Medicine: A Comprehensive Study Guide, 8th Edition. Columbus: McGraw-Hill Education/Medical; 2015.

8. Kieboom JK, et al. Outcome after resuscitation beyond 30 minutes in drowned children with cardiac arrest and hypothermia: Dutch nationwide retrospective cohort study. BMJ. 2015:350. https://doi.org/10.1136/bmj.h418.

\section{Publisher's Note}

Springer Nature remains neutral with regard to jurisdictional claims in published maps and institutional affiliations. 